\title{
Developing Personalized Knowledge Navigation Service for Students Self- Learning based on Interpretive Structural Modeling
}

\author{
${ }^{1}$ Zheng-Hong Wu, ${ }^{2}$ Yu-Jun Liu, ${ }^{3}$ Maiga Chang, ${ }^{4}$ Alex Chang, ${ }^{1}$ Ming Li \\ ${ }^{I}$ School of Information Science \& Technology, East China Normal University, Shanghai 200026, PR. \\ China \\ ${ }^{2}$ Dept. of Educational Technology, Zhejiang University of Technology, Zhejiang 310000, PR. China \\ ${ }^{3}$ Program Office of National Science and Technology Program for e-Learning in Taiwan, Chung- \\ Li,320,Taiwan \\ ${ }^{4}$ Dept. of Information and Computer Engineering, Chung-Yuan Christian University, Taiwan \\ wzh_hongzheng@hotmail.com,walter@zjut.edu.cn,maiga@ms2.hinet.net, \\ akai_aslada@yahoo.com.tw,mli@ee.ecnu.edu.cn
}

\begin{abstract}
This paper designs a personalized navigation service based on the student cognitive levels. The personalized navigation service takes the interpretive structural modeling to generate concept navigation matrix by using the student cognitive background matrix (retrieved from tests and questionnaire) and the concept relation matrix (retrieved from textbooks). According to the knowledge structure and the concept navigation matrix, the service can provide different students their own knowledge navigation maps.

\section{Introduction}

In this paper, we develop a personalized navigation service to generate personal knowledge navigation map for students self-learning in the virtual universities. The service uses interpretive structural modeling (ISM) [1] and knowledge structure to figure out what cognitive level a student achieved and what concepts a student should either learn next or review again. Therefore, a student could realize how much he/she had already learned and which parts (says concepts or instruction units) he/she needs to study again through the visualized knowledge navigation map.

Section 2 gives an overview of the architecture of generating personal knowledge navigation map. The operation flow is also described based on ISM and knowledge structure step by step in Section 2. Finally, Section 3 makes a simple conclusion and describes some possible future works.
\end{abstract}

\section{Personalized Navigation Service}

Figure 1 shows how the personalized knowledge navigation service works.

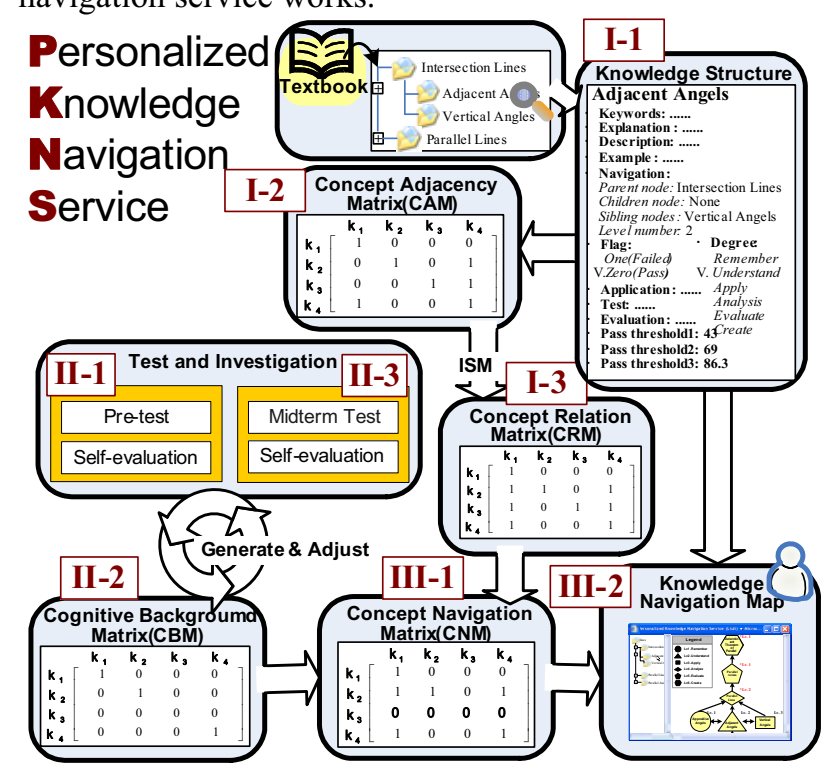

Figure 1. Personalized Knowledge Navigation Service

In the beginning, the service retrieves the knowledge structure from the textbooks (step I-1). Knowledge structure is used to store attributes of concept. There are several attributes in a concept, including keywords, explanation, description, example, navigation, degree, application, test, evaluation, pass threshold, and a flag. Two important parts are navigation and degree which are described within all prescribed textbooks in China now. Navigation part stores the concept relations include parent 
concept (parent node), children concepts (children nodes), and sibling concepts (sibling nodes). Degrees are the cognitive levels proposed by Bloom in 1956 [2]. The student cognitive levels are, from simplest to most complex: remember, understand, apply, analyze, evaluate, and create. The degree indicates the cognitive level of specific concept which students must reach in each instruction unit of textbooks. The flag indicates a student's cognitive level in one concept. The flag value is gotten by testing students. The test scores are divided into six grades corresponding to each of the degrees. A score grade is a flag value from one to six. The dividing criteria are defined by veteran teachers. Evaluation, pass threshold and a flag are also retrieved from the test results. Other attributes are constructed by veteran teachers.

Then, a concept adjacency matrix according to the concept navigation attribute in knowledge structure can be created (step I-2). Warfield (1982) used concept adjacency matrix to represent the direct relationship between two concepts [1]. Finally, the service uses interpretive structural modeling (ISM) to calculate the concept relation matrix (step I-3).

To generate a personal knowledge navigation map, the service gets student cognitive background matrix according to his/her pre-test and self-evaluation results (step II-1 to II-2) or midterm test and self-evaluation results (step II-3 to II-2).

These repeated flows indicate that a student cognitive background matrix would be changed because he/she might reach the level of specific concept during the learning process. According to these test results, we use zero (0) in the cognitive background matrix to indicate that a student reaches the level of specific concept.

The final part, the service generates the concept navigation matrix for specific student according to both the student cognitive matrix (step II-2) and the concept relation matrix (step I-3) as the followings:

Step 1. According to the knowledge structure, the concept adjacency matrix $(C A M)$ can be retrieved as I-2 shows.

Step 2. Defining identity matrix $(I)$ based on the size of concept adjacency matrix according to Warfield's ISM theory.

Step 3. According to ISM, the service has to find the concept relation matrix $\left(C R M=(C A M+I)^{n}\right)$ which satisfies $(C A M+I)^{n-1} \neq(C A M+I)^{n}=$ $(C A M+I)^{n+1}$, as step I-3 shows.

Step4. According to domain experts (teachers), the service makes matrix multiplication between cognitive background matrix $(C B M)$ and concept relation matrix $(C R M)$ to get concept navigation matrix $(C N M), C N M=C B M *$ $C R M$, as step III-1 shows.
If a row value in the concept navigation matrix (step III-1) equals to 0 , then it indicates that the student has reach the learning goal of knowledge point corresponding to this row.

After the concept navigation matrix is found (step III1), the service combines knowledge structure (step I-1) and the navigation matrix (step III-1) to create a visualized knowledge navigation map (step III-2). Students then would be able to see the map by simply using their web browser. On the left panel (step III-2), students can easily select a concept node in an instruction unit (or lesson) to discover their learning performance. The learning performance then comes out on the right panel based on the selected concept. Students can clearly understand their learning states according to different shapes and cognitive levels:

- the bottom concepts are the premise for the top ones;

- the concept with different shapes mean it requires students to reach different cognitive levels;

- and, the black level information on the top-right near the concept indicates that the student's learning performance on the concept. The red level information is to remind student to study this concept again.

\section{Conclusions}

A questionnaire has been implemented about this personalized navigation service for students. The results showed this service is necessary and important to students do self-learning. We should have a more accurate method to reach personalization and adaptively present personalized knowledge navigation map. Furthermore, because the student cognitive level changes dynamically during the self-learning process, we have to improve the cognitive level evaluation method. Besides, we also need to establish standards to judge whether students had reached the concept learning goals or not.

\section{Acknowledgement}

This work was supported in part by the National Natural Science Foundation of China under the project grant number 60573125 .

\section{References}

[1] John N. Warfield, "Interpretive Structural Modeling (ISM)", in S.A. Olsen ed., Group Planning \& Problem Solving Methods in Engineering, 1982, New York: Wiley, 155-201 and 408-411.

[2] B. S. Bloom, M. D. Englehart, E. J. Furst, W. H. Hill, D. R. Krathwohl (Eds.), Taxonomy of educational objectives: The classification of educational goals - Handbook 1: Cognitive Domain, 1956, New York: David McKay. 